\title{
Impact of Time Interval between Trauma Onset and Burr Hole Surgery on Recurrence of Late Subacute or Chronic Subdural Hematoma
}

\author{
Dae-in Kim, M.D., Jae-hoon Kim, M.D., Hee-in Kang, M.D., Byung-gwan Moon, M.D., Joo-seung Kim, M.D., Deok-ryeong Kim, M.D.
}

Department of Neurosurgery, Eulji General Hospital, College of Medicine, Eulji University, Seoul, Korea

\begin{abstract}
Objective : Although subdural hematoma (SDH) is commonly treatable by burr hole surgery in the late subacute or chronic stage, there is no clear consensus regarding appropriate management and exact predictive factors for postoperative recurrence also remain unclear. The aim of this study was to evaluate risk factors associated with recurrence of SDH that requires burr hole surgery in the late subacute or chronic stage. We also identified the appropriate timing of surgery for reducing the recurrence.

Methods : We retrospectively reviewed 274 patients with SDH in the late subacute or chronic stage treated with burr hole surgery in our hospital between January 2007 and December 2014. Excluding patients with acute intracranial complications or unknown time of trauma onset left 216 patients included in the study.

Results : Of 216 patients with SDH in the late subacute or chronic stage, recurrence was observed in 36 patients (16.7\%). The timing of the operation in patients with late subacute stage (15-28 days) resulted in a significant decrease in recurrence (RR, 0.33; 95\% $\mathrm{Cl}, 0.17-0.65 ; p=0.001) \mathrm{com}$ pared to chronic stage (>28 days). Otherwise, no significant risk factors were associated with recurrences including comorbidities and surgical details. Conclusion : The results indicated that time from trauma onset to burr hole surgery may be important for decreasing the risk of recurrence. Therefore, unless patients can be treated conservatively without surgery, prompt surgical management is recommended in patients diagnosed as having late subacute or chronic subdural hematoma treatable by burr hole surgery, even when neurological deficits are unclear.
\end{abstract}

Key Words : Subdural hematoma $\cdot$ Recurrence $\cdot$ Time.

\section{INTRODUCTION}

Subdural hematoma (SDH) in the late subacute or chronic stage is an abnormal collection of viscous liquid or liquefied blood degradation underneath the dura matter ${ }^{2}$. SDH is one of the most common pathologies associated with intracranial hemorrhage and may result in brain tissue compression and neurological sequelae $e^{2,31)}$. SDH in late subacute or chronic stage is increasing in frequency, especially in aging populations as the world's older population rises because of advances in medical care ${ }^{22)}$. In addition, SDH is regarded as a sentinel health event considering its high morbidity and mortality rates in older people ${ }^{10,24)}$. SDH has long been categorized as acute, subacute, or chronic stage based on the period from trauma until symptom onset ${ }^{23}$. Acute subdural hematoma (ASDH) and subacute subdural hematoma (SASDH) or chronic subdural hematoma (CSDH) are different in radiological findings, pathophysiology, surgical management, recurrence, and outcome ${ }^{19,25)}$. Structural alteration of the wall of the hematoma cavity consisting of neomembranes is an important difference ${ }^{41)}$. However, SASDH and CSDH are not clearly distinct in pathophysiology, continuity, or surgical treatment although some studies found that SASDH differs from $\mathrm{CSDH}$ in pathogenesis of hematoma enlargement ${ }^{19)}$. SDH in late subacute or chronic stage is treatable by burr hole surgery. This procedure is a relatively simple, safe, and cost-effective way of managing this condition compared to craniotomy, the risks of which are associated with higher morbidities because it is a comparatively major procedure that uses general anesthesia ${ }^{2)}$. However, although subdural hematoma in late subacute or chronic stage can spontaneously resolve after relieving pressure by draining the liquefied hematoma via burr hole and stabilizing residual incompletely drained hematoma, occasionally SDH recurs. Previous

- Received : March 29, 2016 • Revised : June 14, 2016 •Accepted : July 20, 2016

- Address for reprints : Deok-ryeong Kim, M.D.

Department of Neurosurgery, Eulji General Hospital, College of Medicine, Eulji University, 14 Hangeulbiseok-gil, Nowon-gu, Seoul 01830, Korea

Tel : +82-2-970-8268, Fax : +82-2-979-8268, E-mail : drkdr@hanmail.net

- This is an Open Access article distributed under the terms of the Creative Commons Attribution Non-Commercial License (http://creativecommons.org/licenses/by-nc/3.0) which permits unrestricted non-commercial use, distribution, and reproduction in any medium, provided the original work is properly cited. 
reports found that about $3.7 \%$ to $30 \%$ of patients recur at the same cranial side within a short period ${ }^{4,12,27,28)}$. Many parameters have been suggested as risk factors for recurrence of late subdural or chronic SDH after burr hole surgery. However, no consensus exists on the ability of these factors to predict recurrence ${ }^{15)}$. The aim of this study was to identify the importance of the right timing for surgery to reduce the recurrence of SDH treatable by burr hole surgery in late subacute or chronic stage. We also evaluated risk factors associated with recurrence.

\section{MATREIALS AND METHODS}

\section{Study design and assessment}

A retrospective analysis was performed of 274 patients with $\mathrm{SDH}$ who underwent burr hole surgery in late subacute or chronic stage from January 2007 through December 2014. All cases were confirmed based on computed tomography (CT) results. Symptoms at presentation included headache, limb weakness or altered mentality, altered mentality, and confusion. Within the group of 274274 patients, we excluded patients with unknown trauma onset time, selection of craniotomy as main treatment modality and acute intracranial complications such as acute epidural or SDH development after burr hole surgery. Ultimately, 216 patients were included and arranged into two groups. Group 1 contained 116 patients who underwent surgery in the late subacute stage with a 3- to 4-week time interval between trauma onset and operation. Group 2 contained 100 patients treated in the chronic stage after 4 weeks after trauma onset (Fig. 1). With approval from the hospital ethics committee, we reviewed medical records and radiology for all patients. The following parameters were recorded and analyzed : sex; age at surgery; medical history such as diabetes mellitus, hypertension, liver disease, renal disease, cancer, stroke, or ischemic heart disease; recent use of antiplatelet or anticoagulant therapy; total amount of hematoma drainage; number of days until drain catheter removal; and recurrence. All preoperative and postoperative CT scans were retrospectively reviewed by two neurosurgeons. CSDH was classified into three types according to density on CT scan : hypodensity ( $<25 \mathrm{HU})$, isodensity $(25-35 \mathrm{HU})$, and mixed type ${ }^{33)}$. Mass effect defined as a midline deviation of the septum pellucidum and pineal body was measured at the level of the foramen of Monro on preoperative CT scans. Air collection was categorized into groups according to subdural air found on immediate postoperative CT scan. The none or mild group had residual air bubbles; the definite group had near total replacement with air $^{29)}$. Recurrence was defined as radiological and clinical findings warranting further treatment on the ipsilateral side. All patients were followed up for at least 6 months.

\section{Surgical management}

In our department, burr hole surgery was the treatment of choice for patients with symptomatic SDH in the late subacute or chronic stage. Details of the perioperative techniques differed little among individual surgeons. Most patients underwent one burr hole craniostomy, hematoma evacuation with saline irrigation to facilitate rinsing the hematoma, and closed system drainage with meticulous avoidance of air entry under local anesthesia. Postoperatively, patients were kept in bed rest following surgical drainage to enhance gravitational drainage of residual subdural fluid. Adequate hydration was given for 3-4 days to promote expansion of the brain. The drain was usually maintained for two additional days (up to 7 days) postoperatively and removed after CT scan when drainage became negligible.
Fig. 1. Flowchart of the study population.

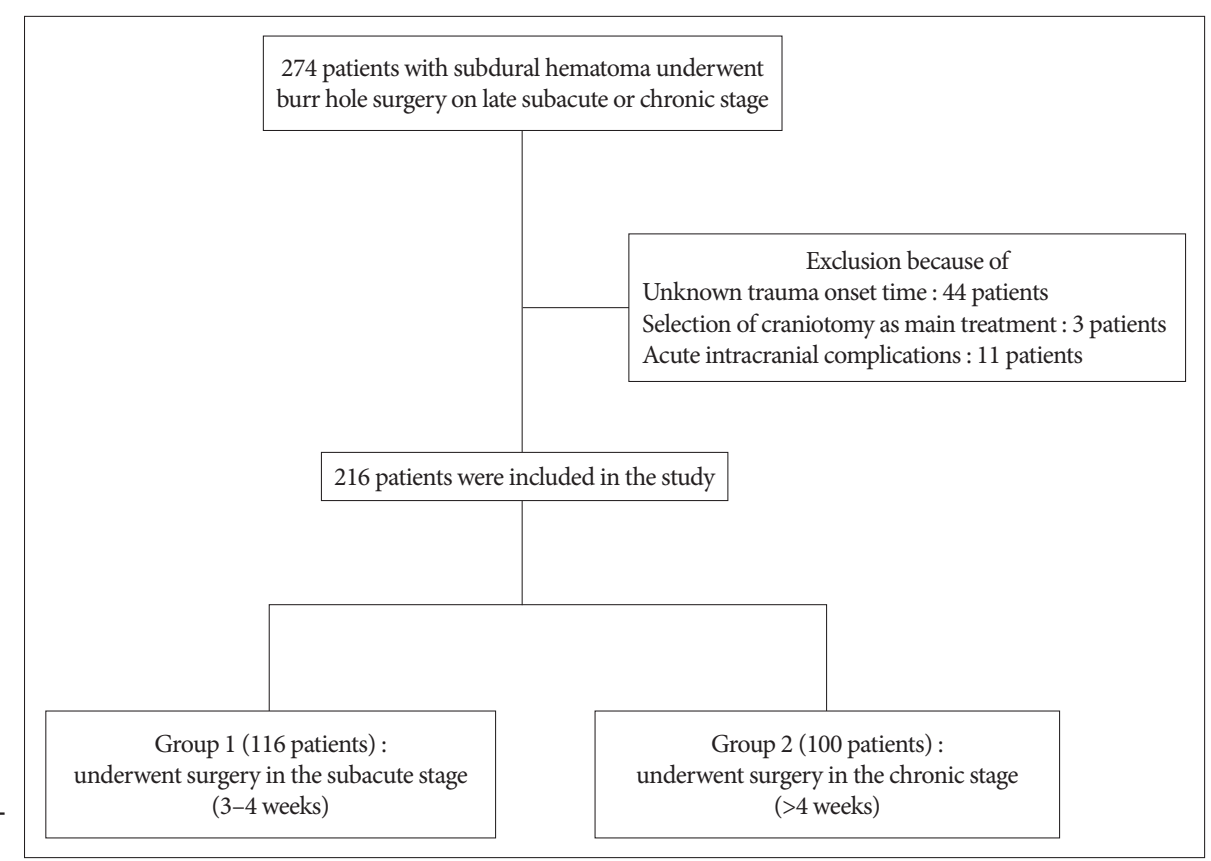




\section{Statistical analysis}

Comparisons between groups were analyzed using the chisquare test, Fisher's exact test, and Student's t-test. Data are presented as mean \pm standard deviation. Multivariate analysis using a logistic regression model controlled for age, sex, CT density, antiplatelet medication, postoperative midline displacement and air collection to evaluate association between time interval from trauma onset until operation and recurrence. Odds ratios, $95 \%$ confidence intervals, and $p$ values of covariates were reported. All statistical tests were two-sided, and $p<0.05$ was considered statistically significant. Analyses were performed using the statistical software SPSS (ver. 17.0; SPSS Inc., Chicago, IL, USA).

\section{RESULTS}

\section{Characteristics of the study population}

At surgery, patients were mean $70 \pm 12.6$ years old (range 33-
92 years); 142 were men (66\%). The average number of comorbidities was $1.44 \pm 1.52$ (range $0-5$ ). There were bilateral hematomas in 26 patients (12\%); these were excluded in analysis of midline deviation. Patients were divided into two groups according to operation time from trauma onset. One group was patients treated in the late subacute stage and the other was patients treated in the chronic stage. Clinical characteristics of the groups are in Table 1. In group 1, patients treated in late subacute stage, mean age was $68.9 \pm 11.87$ years, 80 patients were men (69\%), 68 patients had hypertension, 40 had diabetes mellitus, 11 had liver cirrhosis, 7 had chronic kidney disease, 6 had a malignancy, 16 had cerebrovascular disease, and 13 had ischemic heart disease. In this group, 28 patients (24\%) had taken antiplatelet medication. In group 2, patients treated in chronic stage, the mean age was $70.4 \pm 12.62$ years, 62 were men (62\%), 56 patients had hypertension, 42 had diabetes mellitus, 9 had liver cirrhosis, 12 had chronic kidney disease, 3 had a malignancy, 21 had cerebrovascular disease, and 9 had ischemic heart disease. In this group,

Table 1. Baseline characteristics of the patients

\begin{tabular}{|c|c|c|c|c|}
\hline & Group $1(n=116)$ & Group $2(n=100)$ & Total (216) & $p$ value \\
\hline Sex & & & & 0.282 \\
\hline Male & 80 & 62 & 142 & \\
\hline Female & 36 & 38 & 74 & \\
\hline Age & & & & 0.369 \\
\hline Mean & 68.88 & 70.38 & & \\
\hline $\mathrm{SD}$ & 11.865 & 12.628 & & \\
\hline \multicolumn{5}{|l|}{ Comorbidities } \\
\hline Hypertension & 68 & 56 & & 0.698 \\
\hline Diabetes mellitus & 40 & 42 & & 0.2561 \\
\hline Liver cirrhosis & 11 & 9 & & 0.903 \\
\hline Chronic renal failure & 7 & 12 & & 0.123 \\
\hline Cancer & 6 & 3 & & 0.510 \\
\hline Stroke & 16 & 21 & & 0.222 \\
\hline Ischemic heart disease & 13 & 9 & & 0.593 \\
\hline \multicolumn{5}{|l|}{ Medication } \\
\hline Antiplatelet & 28 & 34 & & 0.11 \\
\hline Anticoagulants & 4 & 2 & & 0.688 \\
\hline \multicolumn{5}{|l|}{ Preoperative CT scan findings } \\
\hline Density & & & & 0.000 \\
\hline Iso & 89 & 18 & & \\
\hline Low & 22 & 71 & & \\
\hline Mixed & 5 & 11 & & \\
\hline Midline deviation (in unilateral SDH) & $9.78 \pm 4.534(108)$ & $7.06 \pm 4.924(82)$ & 190 & 0.000 \\
\hline Postoperative air collection & & & & 0.413 \\
\hline None or mild & 106 & 88 & & \\
\hline Definite & 10 & 12 & & \\
\hline \multicolumn{5}{|l|}{ Surgical details } \\
\hline Mean total amount of drainage $(\mathrm{mL})$ & $144.40 \pm 98.442$ & $161.85 \pm 142.445$ & & 0.304 \\
\hline Mean No. of days until drain removal & $3.71 \pm 1.258$ & $3.61 \pm 1.230$ & & 0.097 \\
\hline Recurrence & & & & 0.001 \\
\hline No & 106 & 74 & 180 & \\
\hline Yes & 10 & 26 & 36 & \\
\hline
\end{tabular}

SD : standard deviation, CT : computed tomography, SDH : subdural hematoma 
34 patients (34\%) had taken antiplatelet medication. Comparison of radiological factors and surgical details between the two groups is in Table 1. For group 1, SDH was isodense in 89 patients, hypodense in 22, and mixed in 5 . Mean midline displacement in unilateral SDH patients was $9.78 \pm 4.53 \mathrm{~mm}$, air collection on immediate postoperative CT scan was mild in 106 patients and definite in 10. Mean total hematoma drainage amount was 144.4. $\pm 98.42 \mathrm{~mL}$ and mean duration prior to drain removal was $3.71 \pm 1.25$ days. For group 2, SDH was isodense in 18 patients, hypodense in 71 , and mixed in 11 . The mean midline displacement in unilateral SDH patients was $7.06 \pm 4.92 \mathrm{~mm}$, air collection on immediate postoperative CT scan was mild in 88 patients and definite in 12. Mean total hematoma drainage amount was $161.85 . \pm 142.45 \mathrm{~mL}$ and mean duration prior to drain removal was $3.61 \pm 1.23$ days. No differences between groups in sex, age, comorbidity, antiplatelet or anticoagulant medication, or surgical details were significant. However, recurrence rate, was $8.6 \%$ (10 patients) for group 1 and $26 \%$ (26 patients) for group 2 , significant difference $(p=0.001)$. Differences were also significant for preoperative CT density and midline deviation.

\section{Factors predicting recurrence}

A total of 36 patients ( 28 men and 8 women; mean age at surgery $71 \pm 9.8$ years) underwent a second burr hole surgery because of SDH recurrence after initial surgery. Univariate and multivariate logistic regression models predicting recurrence are in Table 2. Univariate analysis indicated that preoperative CT density $(p=0.021)$ and time interval $(p=0.001)$ were risk factors for recurrence. After adjusting for confounding factors of age, sex, CT density, antiplatelet medication, postoperative midline dis-

Table 2. Logistic regression analyses predicting recurrence for all 216 patients

\begin{tabular}{|c|c|c|c|c|c|c|}
\hline \multirow{2}{*}{ Variables } & \multicolumn{3}{|c|}{ Crude } & \multicolumn{3}{|c|}{ Adjusted } \\
\hline & OR & $95 \% \mathrm{CI}$ & $p$-value & OR & $95 \% \mathrm{CI}$ & $p$-value \\
\hline \multicolumn{7}{|l|}{ Age (years) } \\
\hline$<70$ & 1.000 & & 0.171 & 1.000 & & 0.119 \\
\hline$\geq 70$ & 1.733 & $0.788-3.811$ & & 2.271 & $0.810-6.367$ & \\
\hline \multicolumn{7}{|l|}{ Sex } \\
\hline Female & 1.000 & & 0.1 & 1.000 & & 0.036 \\
\hline Male & 2.026 & $0.873-4.704$ & & 2.908 & $1.072-7.890$ & \\
\hline \multicolumn{7}{|l|}{ CT density } \\
\hline Iso & 1.000 & & 0.021 & 1.000 & & 0.943 \\
\hline Low & 2.545 & $1.154-5.614$ & & 1.044 & $0.322-3.377$ & \\
\hline \multicolumn{7}{|l|}{ Diabetes mellitus } \\
\hline No & 1.000 & & 0.381 & & & \\
\hline Yes & 1.382 & $0.670-2.850$ & & & & \\
\hline \multicolumn{7}{|l|}{ Hypertension } \\
\hline No & 1.000 & & 0.623 & & & \\
\hline Yes & 1.202 & $0.578-2.499$ & & & & \\
\hline \multicolumn{7}{|l|}{ Antiplatelet medication } \\
\hline No & 1.000 & & 0.502 & 1.000 & & 0.851 \\
\hline Yes & 1.300 & $0.604-2.796$ & & 0.908 & $0.334-2.472$ & \\
\hline \multicolumn{7}{|l|}{ Liver cirrhosis } \\
\hline No & 1.000 & & 0.675 & & & \\
\hline Yes & 1.281 & $0.402-4.084$ & & & & \\
\hline \multicolumn{7}{|l|}{ Chronic renal failure } \\
\hline No & 1.000 & & 0.592 & & & \\
\hline Yes & 1.375 & $0.428-4.413$ & & & & \\
\hline \multicolumn{7}{|c|}{ Postoperative air collection } \\
\hline Minimal & 1.000 & & 0.841 & 1.000 & & 0.851 \\
\hline Definite & 1.125 & $0.357-3.545$ & & 0.765 & $0.204-2.874$ & \\
\hline \multicolumn{7}{|l|}{ Midline deviation } \\
\hline$<10$ & 1.000 & & 0.796 & 1.000 & & 0.286 \\
\hline$\geq 10$ & 1.111 & $0.501-2.467$ & & 1.639 & $0.661-4.603$ & \\
\hline \multicolumn{7}{|l|}{ Time interval } \\
\hline 3-4 weeks & 1 & $1.695-8.185$ & & 1.000 & & $0.028^{\star}$ \\
\hline$>4$ weeks & 3.274 & & 0.001 & 3.543 & $1.144-10.976$ & \\
\hline
\end{tabular}

${ }^{*}$ Adjusted by age, sex, CT density, antiplatelet medication, postoperative midline displacement, and air collection. CT : computed tomography 
placement, and air collection, only time interval was associated with increased risk of recurrence (adjusted odds ratio 3.54, 95\% confidence interval $1.144-10.976, p=0.028$ ). To identify factors independently associated with recurrence, including time interval, differences in all available covariates were examined. Male sex was associated with significantly increased recurrence.

\section{DISCUSSION}

A conventionally used classification divides subdural hematomas into acute, subacute, and chronic according to time between injury and onset of clinical symptoms. Time intervals are divided into acute for presentation with urgent symptoms within three days of trauma, subacute for presentation within 4 to 21 days, and chronic for presentation after 21 days. The conventional classification of SDH is widely accepted and generally applied without controversy. However, we found that this classification is based on a publication from 1960 by McKissock et al. ${ }^{23)}$ designed to consider symptom evolution related to prognosis and time. The characteristics of blood in imaging such as CT or magnetic resonance imaging (MRI) do not affect the classification because these methods were not commercialized at the time of the publication. In practice, in patients with $\mathrm{SDH}$, imaging to determine the type of hematoma is useful for diagnosing the changing state of SDH and for surgical decision-making with the time between the injury and the onset of neurological symptoms. Fujisawa et al. ${ }^{11)}$ assumed that hematoma stability varies depending on the stage with some correlation between hematoma stage and radiological findings. On the basis of CT presentation, hyperdense hematoma is classified as acute, isodense as subacute, and hypodense as chronic ${ }^{13)}$. To date, most studies have considered division of SDH by time course of development to be closely linked to CT-based division and often use them interchangeably. However, the divisions are frequently inconsistent. In the presumed CSDH phase, persistence of isodensity on CT scan or high signal intensity on T1-weighted MRI, typical of subacute intracranial hematomas beyond the expected time inter$\mathrm{val}$, is common and responsible for confusion. Godlewski et al. ${ }^{13)}$ reported classifying SDH as acute, subacute, and chronic according to widely accepted divisions based on CT presentation and patients with chronic hematomas did not differ significantly from patients with subacute hematomas in time from injury to onset of symptoms. Isodense SDH lesions in $\mathrm{CSDH}$ patients who are conventionally classified are often reported as more common than hypodense lesions on CT scans ${ }^{33)}$. The appearance on MRI of most brain lesions is directly correlated with $\mathrm{CT}^{5)}$. T1 shortening, which results in hyperintensity, generally represents methemoglobin. The typical component of subacute hematoma and chronic hematoma may be slightly hypointense relative to grey matter on T1-weighted images ${ }^{6,14)}$. Despite substantial variability, the five stages of subdural hematoma and intracerebral hemorrhage (ICH) are generally accepted to be distinguishable according to characteristic appearances on T1-weighted and T2- weighted MRI. The stages, based on the period between MRI examination and start of bleeding, are classified as hyperacute (within 24 hours), acute (from 24 hours to up to 3 days), early subacute (3-7 days), late subacute (from 8 days to 1 month), or chronic (more than 1 month) $)^{5,16,32)}$. SDH evolves more slowly than ICH because the oxygen tension in these compartments is higher ${ }^{6}$. SASDH, which represents evolving hematomas as liquefaction of the initial clot, causes symptomatic expansion with the beginning of liquefaction at 2 weeks after hematoma formation $^{17)}$. Thus, burr hole surgery with hematoma drainage for SASDH or CSDH might be advantageous when performed at least 2 weeks after trauma onset and the time interval classifying the chronic stage could be moved back by a week based on relationship with radiological findings. For this reason we suggest revising the late subacute stage to 3 to 4 weeks after trauma onset and revising the chronic stage as after 4 weeks from trauma onset.

$\mathrm{SDH}$ in the late subacute or chronic stage can be conveniently evacuated by burr hole surgery. These hematomas are sufficiently liquefied and mostly being as minimal or insignificant ASDH, aggravated mostly in the subacute phase ${ }^{18)}$. SASDH is reported to occur in approximately $10 \%$ of acute SDH cases that are treated conservatively ${ }^{18,30)}$. Multiple factors are responsible for enlargement of hematomas over time. Hemodynamically, two mechanisms have been suggest : invasion of cerebrospinal fluid by a difference in osmotic pressure and cerebral hyperperfusion that follows hypoperfusion of the acute phase ${ }^{3,35,37)}$. Pathophysiologically, following an initial head injury, blood within the subdural space triggers an inflammatory response that leads to high concentrations of vascular endothelial growth factor, increasing the permeability of capillaries ${ }^{38)}$. After approximately two weeks, neomembranes on the inner and outer surface are formed through dural collagen synthesis and fibroblast spread, followed by ingrowth of neocapillaries, enzymatic fibrinolysis, and hematoma liquefaction ${ }^{8,934)}$. Fibrin degradation products are reincorporated into new clots and inhibit hemostasis ${ }^{26)}$. The clinical course of hematoma evolution is determined by a combination of recurrent episodes of inflammation, microbleeding and hyperfibrinolysis. SASDH is a transitional state showing a transformation from ASDH to CSDH. An important feature of SASDH that is considered a distinct pathophysiological entity is an immature outer membrane differing from the hemorrhagic outer membrane of $\mathrm{CSDH}^{19)}$. Based on ultrastructural observations, ongoing angioneogenetic activity of hematoma cavity wall consisting of neomembranes is accepted to be part of the course of chronification $^{41)}$. The outer membrane displays signs of marked pathological vascularization compared with the almost avascular inner membrane ${ }^{41}$. The vessels of the neomembrane have a marked proliferation potential and are fragile, leading to repeated microhemorrhaging from the neocapillary network and fibrinolytic activity of fibrinogen degradation products; this is the most important factor for gradual hematoma growth without coagulation ${ }^{20,26,40)}$. Izumihara et al. ${ }^{19)}$ report- 
ed 8 operative cases of SDH in the late subacute stage (15-23 days) and found thin outer membranes differing from the mature hemorrhagic outer membrane of CSDH in most patients (7 patients). In their study, SASDH was considered to differ from $\mathrm{CSDH}$ not only based on the characteristic operative and histological findings, but also in that none of the $8 \mathrm{SASDH}$ patients required reoperation. Another study by Kpelao et al. ${ }^{21)}$ defined SASDH as cranial trauma between 8 and 21 days associated with isodensity on CT. In that study, SASDH did not have a neomembrane intraoperatively, unlike CSDH. In a study by Godlewski et al. ${ }^{13)}$, reoperation following burr hole surgery as the primary procedure for hematoma evacuation was significantly more frequent in patients with chronic hematomas compared to those with subacute hematoma. Therefore, ongoing angioneogenesis in the neomembrane (especially, the outer membrane) constituting the hematoma cavity is strongly regarded to be a salient factor in successive hematoma enlargement and recurrence. Progression of the condition needs to be prevented early by draining the bulk of the hematoma, which has a high concentration of multiple growth factors for forming new blood vessels. Our study compared the recurrence between late subacute stage and chronic stage. We found that recurrence of patients treated in the late subacute stage was significantly lower than for patients with chronic hematomas $(p=0.001)$. Previously published studies and our study support the hypothesis that time from trauma onset to burr hole surgery is important for predicting risk of recurrence.

Recurrence is the most common complication following burr hole surgery of liquefied SDH with a median incidence of $15 \%$ in the literature ${ }^{4,12,27,28)}$. Various surgical techniques have been used to decrease recurrence and many risk factors for recurrence have been investigated. Clinical factors thought to be associated with higher recurrence rates, although disputed, include age, diabetes mellitus, alcoholism, cerebral atrophy, antiplatelet and anticoagulant drug use, poor performance status on admission, poor Glasgow coma scale and Glasgow outcome scale, bilateral lesion, depth of hematoma, postoperative midline displacement, postoperative subdural air collection, septum formation or multiple membranes in the hematoma cavity, higher hematoma density on CT, and hematoma with laminar and separated architecture $\mathrm{1}^{1,7,12,28,31,39,42)}$. A meta-analysis of 5 randomized trials indicated a significant reduction in recurrence when drains were inserted following hematoma evacuation ${ }^{2,36)}$. A preoperative MRI finding showed that a T1-iso/hypointensity group had significantly higher risk of recurrence relative to a T1-hyperintensity group $^{15)}$. This study suggested that hematoma stability varies depending on stage and T1-hyperintensity hematomas are more stable than those in the T1-iso/hypointensity group. Our logistic regression analysis showed that time was a significant predictor of recurrence. The recurrence for patients with a time interval corresponding to late subacute stage was significantly lower than for patients with chronic stage T1-hyperintensity hematoma was mostly observed in the subacute stage. Consequently, these MRI results appear to confirm our findings regarding the stability of subacute stage hematomas and appropriate surgical timing.

This study had limitations mostly stemming from the retrospective design. Methodological weaknesses included : 1) retrospectively collected data could be missing or misinterpreted;2) inevitable heterogeneity between groups in other disease characteristics and clinical factors could affect outcomes; and 3) detailed differences among management options including variable surgical techniques, different instruments, and advances in imaging techniques. These limitations reduce ability to compare chronification between different hematoma stages and impair the precise assessment of lesion progression over time. Even though burr hole surgery under local anesthesia was the treatment of choice in this study, details of the perioperative techniques such as the number of burr holes, irrigation of the subdural space, use of drains after burr hole evacuation, and staging of bed rest following surgery varied by surgeon; these factors thus represent the possibility of bias. To effectively address the causal relationships suggested by this study, future research with randomized controlled trials with well-defined protocols are required.

\section{CONCLUSION}

The results of this study suggested that time from trauma onset to burr hole surgery in patients with SDH in the late subacute or chronic stage may be important in decreasing recurrence risk. Accordingly, patients diagnosed with late SASDH or CSDH that is treatable by burr hole surgery should not be deferred when an operation is required even though neurological deficits are unclear.

\section{References}

1. Abouzari M, Rashidi A, Rezaii J, Esfandiari K, Asadollahi M, Aleali H, et al. : The role of postoperative patient posture in the recurrence of traumatic chronic subdural hematoma after burr-hole surgery. Neurosurgery 61 : 794-797; discussion 797, 2007

2. Almenawer SA, Farrokhyar F, Hong C, Alhazzani W, Manoranjan B, Yarascavitch B, et al. : Chronic subdural hematoma management : a systematic review and meta-analysis of 34,829 patients. Ann Surg 259 : 449-457, 2014

3. Aoki N, Tsutsumi K : Symptomatic subacute subdural haematoma following spontaneous acute subdural haematoma. Acta Neurochir (Wien) 102 : 149-151, 1999

4. Baechli H, Nordmann A, Bucher HC, Gratzl O : Demographics and prevalent risk factors of chronic subdural haematoma : results of a large single-center cohort study. Neurosurg Rev 27 : 263-266, 2004

5. Bradley WG Jr : MR appearance of hemorrhage in the brain. Radiology 189 : 15-26, 1993

6. Bradley WG Jr, Schmidt PG : Effect of methemoglobin formation on the MR appearance of subarachnoid hemorrhage. Radiology 156 : 99103, 1985

7. Chon KH, Lee JM, Koh EJ, Choi HY : Independent predictors for recurrence of chronic subdural hematoma. Acta Neurochir (Wien) 154 : 1541 1548, 2012

8. Drapkin AJ : Chronic subdural hematoma : pathophysiological basis for treatment. Br J Neurosurg 5 : 467-473, 1991 
9. Ducruet AF, Grobelny BT, Zacharia BE, Hickman ZL, DeRosa PL, Andersen KN, et al. : The surgical management of chronic subdural hematoma. Neurosurg Rev 35 : 155-169; discussion 169, 2012

10. Dumont TM, Rughani AI, Goeckes T, Tranmer BI : Chronic subdural hematoma : a sentinel health event. World Neurosurg 80 : 889-892, 2013

11. Fujisawa H, Nomura S, Kajiwara K, Kato S, Fujii M, Suzuki M : Various magnetic resonance imaging patterns of chronic subdural hematomas : indicators of the pathogenesis? Neurol Med Chir (Tokyo) 46 : 333-338; discussion 338-339, 2006

12. Gelabert-González M, Iglesias-Pais M, García-Allut A, Martínez-Rumbo $\mathrm{R}$ : Chronic subdural haematoma : surgical treatment and outcome in 1000 cases. Clin Neurol Neurosurg $107:$ 223-229, 2005

13. Godlewski B, Pawelczyk A, Pawelczyk T, Ceranowicz K, Wojdyn M, Radek M : Retrospective analysis of operative treatment of a series of 100 patients with subdural hematoma. Neurol Med Chir (Tokyo) 53 : 26-33, 2013

14. Gomori JM, Grossman RI : Mechanisms responsible for the MR appearance and evolution of intracranial hemorrhage. Radiographics 8 : 427440, 1988

15. Goto H, Ishikawa O, Nomura M, Tanaka K, Nomura S, Maeda K : Magnetic resonance imaging findings predict the recurrence of chronic subdural hematoma. Neurol Med Chir (Tokyo) 55 : 173-178, 2015

16. Hayman LA, Taber KH, Ford JJ, Bryan RN : Mechanisms of MR signal alteration by acute intracerebral blood : old concepts and new theories. AJNR Am J Neuroradiol 12 : 899-907, 1991

17. Huang KT, Bi WL, Abd-El-Barr M, Yan SC, Tafel IJ, Dunn IF, et al. : The Neurocritical and neurosurgical care of subdural hematomas. Neurocrit Care $24: 294-307,2016$

18. Izumihara A, Orita T, Tsurutani T, Kajiwara K : [Natural course of nonoperative cases of acute subdural hematoma : sequential computed tomographic study in the acute and subacute stages]. No Shinkei Geka 25 : 307-314, 1997

19. Izumihara A, Yamashita K, Murakami T : Acute subdural hematoma requiring surgery in the subacute or chronic stage. Neurol Med Chir (Tokyo) $53: 323-328,2013$

20. Kawakami Y, Chikama M, Tamiya T, Shimamura Y : Coagulation and fibrinolysis in chronic subdural hematoma. Neurosurgery $25: 25-29,1989$

21. Kpelao E, Beketi KA, Moumouni AK, Doleagbenou A, Ntimon B, Egbohou P, et al. : Clinical profile of subdural hematomas : dangerousness of subdural subacute hematoma. Neurosurg Rev 39 : 237-240; discussion 240,2016

22. Lee L, Ker J, Ng HY, Munusamy T, King NK, Kumar D, et al. : Outcomes of chronic subdural hematoma drainage in nonagenarians and centenarians : a multicenter study. J Neurosurg 124 : 546-551, 2016

23. McKissock W, Richardson A, Bloom WH : Subdural haematoma : a review of 389 cases. Lancet 275 : 1365-1369, 1960

24. Miranda LB, Braxton E, Hobbs J, Quigley MR : Chronic subdural hematoma in the elderly : not a benign disease. J Neurosurg 114 : 72-76, 2011

25. Morinaga K, Matsumoto Y, Omiya N, Mikami J, Ueda M, Sato H, et al. : [Subacute subdural hematoma--report of 4 cases and a review of the literature]. No To Shinkei 42: 131-136, 1990

26. Murakami H, Hirose Y, Sagoh M, Shimizu K, Kojima M, Gotoh K, et al. : Why do chronic subdural hematomas continue to grow slowly and not coagulate? Role of thrombomodulin in the mechanism. J Neurosurg 96 : 877-884, 2002

27. Nakaguchi H, Tanishima T, Yoshimasu N : Factors in the natural history of chronic subdural hematomas that influence their postoperative recurrence. J Neurosurg 95 : 256-262, 2001

28. Ohba S, Kinoshita Y, Nakagawa T, Murakami H : The risk factors for recurrence of chronic subdural hematoma. Neurosurg Rev 36 : 145-149; discussion 149-150, 2013

29. Oishi M, Toyama M, Tamatani S, Kitazawa T, Saito M : Clinical factors of recurrent chronic subdural hematoma. Neurol Med Chir (Tokyo) 41 : 382-386, 2001

30. Okumura Y, Shimomura T, Park YS : [A study of acute subdural hematoma developing into hematoma with capsule formation]. No Shinkei Geka 26 : 691-698, 1998

31. Pang CH, Lee SE, Kim CH, Kim JE, Kang HS, Park CK, et al. : Acute intracranial bleeding and recurrence after bur hole craniostomy for chronic subdural hematoma. J Neurosurg $123: 65-74,2015$

32. Parizel PM, Makkat S, Van Miert E, Van Goethem JW, van den Hauwe L, De Schepper AM : Intracranial hemorrhage : principles of CT and MRI interpretation. Eur Radiol 11 : 1770-1783, 2001

33. Park HR, Lee KS, Shim JJ, Yoon SM, Bae HG, Doh JW : Multiple densities of the chronic subdural hematoma in CT scans. J Korean Neurosurg Soc 54 : 38-41, 2013

34. Sajanti J, Majamaa K : High concentrations of procollagen propeptides in chronic subdural haematoma and effusion. J Neurol Neurosurg Psychiatry $74: 522-524,2003$

35. Salvant JB Jr, Muizelaar JP : Changes in cerebral blood flow and metabolism related to the presence of subdural hematoma. Neurosurgery 33 : 387-393; discussion 393, 1993

36. Santarius T, Kirkpatrick PJ, Ganesan D, Chia HL, Jalloh I, Smielewski P, et al. : Use of drains versus no drains after burr-hole evacuation of chronic subdural haematoma : a randomised controlled trial. Lancet 374 : 1067-1073, 2009

37. Son S, Yoo CJ, Lee SG, Kim EY, Park CW, Kim WK : Natural course of initially non-operated cases of acute subdural hematoma : the risk factors of hematoma progression. J Korean Neurosurg Soc 54 : 211-219, 2013

38. Tao Z, Ding S, Hu M, Li J : Delayed hypersensitive process in subacute subdural hematoma. J Craniofac Surg 26 : e443-e445, 2015

39. Torihashi K, Sadamasa N, Yoshida K, Narumi O, Chin M, Yamagata S : Independent predictors for recurrence of chronic subdural hematoma : a review of 343 consecutive surgical cases. Neurosurgery 63 : 1125-1129; discussion 1129, 2008

40. Vaquero J, Zurita M, Cincu R: Vascular endothelial growth-permeability factor in granulation tissue of chronic subdural haematomas. Acta Neurochir (Wien) 144: 343-346; discussion 347, 2002

41. Weigel R, Schilling L, Schmiedek P : Specific pattern of growth factor distribution in chronic subdural hematoma (CSH) : evidence for an angiogenic disease. Acta Neurochir (Wien) $143: 811-818$; discussion 819, 2001

42. Yamamoto H, Hirashima Y, Hamada H, Hayashi N, Origasa H, Endo S : Independent predictors of recurrence of chronic subdural hematoma : results of multivariate analysis performed using a logistic regression model. J Neurosurg 98 : 1217-1221, 2003 